\title{
Study on State Estimator for Wave-piercing Catamaran Longitudinal Motion Based on EKF
}

\author{
SHAO Qiwen", *LIU Sheng ${ }^{1}$, WANG Wugui ${ }^{2}$ \\ ${ }^{1}$ Harbin Engineering University, Harbin, 150001 \\ ${ }^{2}$ China Ship Development and Design Center , Wuhan, 4300642) \\ liu.sch@163.com
}

Keywords: EKF; Wave-piercing Catamaran; Longitudinal Motion; State Estimator.

Abstract. For the characteristics of wave-piercing catamaran longitudinal motion when sailing with high speed in random waves, this paper proposes a state estimation algorithm for wave-piercing catamaran longitudinal motion based on EKF. On the one hand noise is filtered out from the noise pollution measurement signal as much as possible, and the desired signal is separated therefrom; on the other hand, all state information of the system can be obtained by simply selecting the pitch angle and heave displacement of wave-piercing catamaran. Theoretical analysis and simulation results show that the proposed state estimation algorithm can achieve satisfactory filtering accuracy and filtering efficiency..

\section{Introduction}

Wave-piercing catamaran is high performance compound ship which is combined with structure and characteristics of ordinary catamaran and SWATH in 1980s. The ship type has the advantages of fast speed, high seakeeping and comfortable which ordinary catamaran can't compare with, and thus once available it has been rapidly promoted and widely applied in various fields [1]. However, wave-piercing catamaran is vulnerable to outside interference such as random waves when sailing at high speed, so that the longitudinal motion is violent [2]. At present, many researchers have begun to study on wave-piercing catamaran longitudinal motion control system at home and abroad. However, in engineering applications, on the one hand some or all states of many systems (including linear systems and nonlinear systems) can't be measured directly, but in order to control effectively, system state information must be obtained in real time; on the other hand, the structural parameters of some or all of the described system is unknown or uncertain. Therefore, the unknown structure parameters and unmeasured states can be estimated by measuring the state which can be measured $\left[3^{][4]}\right.$.

Firstly, the mathematical model of wave-piercing catamaran longitudinal motion is established based on MMG theory. Mathematical model will be converted into state-space equation by simplifying the model to some extent and definition of state variables. It is taken into account that the wave-piercing catamaran often sails in high speed in the sea with random disturbances, and the measuring sensor inevitably exists random noise, and even parts of the system state isn't possible to measure directly. This paper proposes a state estimation algorithm for wave-piercing catamaran longitudinal motion based on EKF, and the feedback 
state amount estimation technique of wave-piercing catamaran longitudinal motion control system is studied. The effectiveness of the proposed algorithm is verified through analyze of theory simulation research.

\section{Establishment of Mathematics Model}

When studying the wave-piercing catamaran longitudinal motion of two degrees of freedom on the random sea, two coordinate systems are generally used, and one is inertial coordinate system fixed on the ground, the other is the body-fitted coordinate system fixed on the wave-piercing catamaran. In this paper, nonlinear model of wave-piercing catamaran longitudinal motion is built by using MMG school separated model, and the wave-piercing catamaran body-fitted coordinate system is shown in Figure 1.

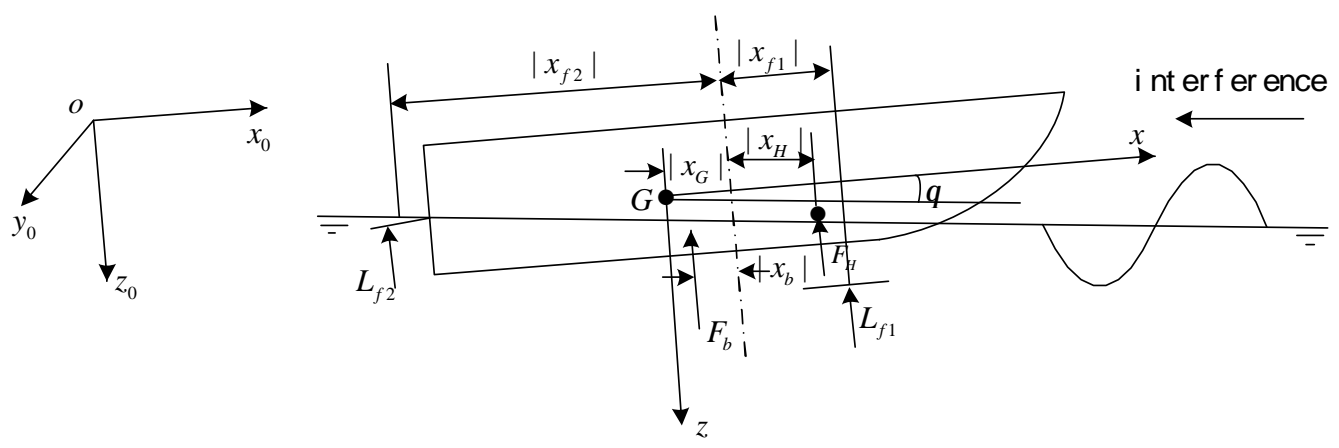

Fig. 1 wave-piercing catamaran body-fitted coordinate system

The wave-piercing catamaran hull is regarded as a rigid body, the longitudinal motion is mainly influenced by force and torque generated by the hull, force and torque generated by hydrofoil, control force and torque generated by breasted tail and stern flap and interference force and torque generated by random waves, etc. And also the effect of the wave-piercing catamaran pitching and motion caused by random waves are considered. Based on rigid body motion momentum theorem and momentum moment theorem with respect to the rigid body motion, the mathematics model of wave-piercing catamaran longitudinal motion control system is as follows:

$$
\left\{\begin{array}{l}
m\left(\llcorner \&-q u)=2 \sum_{i=1}^{2} F_{f i}+2 F_{f p}+2 F_{H}+F_{b} \cos \theta+m g \cos \theta+Z_{1}\right. \\
I_{y y} \&=-2 \sum_{i=1}^{2}\left(x_{f i}-x_{G}\right) F_{f i}-2\left(x_{f p}-x_{G}\right) F_{f p}-2\left(x_{H}-x_{G}\right) F_{H}-\left(x_{b}-x_{G}\right) F_{b} \cos \theta+M_{1}
\end{array}\right.
$$


Two degrees of freedom motion of wave-piercing catamaran which contains heave velocity $w$ and pitch angular velocity $q$ in the body-fitted coordinate system can be converted to the inertial coordinate system by the coordinate transformation which contains heave amount derivative \& and wave-piercing catamaran pitching angle derivative $\theta$. And Ignoring roll and yaw effect, the relationship between them is:

$$
\begin{aligned}
& \delta=-\sin \theta u+\cos \theta w \\
& \theta=q
\end{aligned}
$$

The force and torque coefficient calculating method in formulation (1) is shown in the paper [5].

According to the force calculating method of wave-piercing catamaran, formula (1) can be arranged as the following forms:

$$
\left\{\begin{array}{l}
x=f(x)+g(x) u \\
y_{1}=z_{0} \\
y_{2}=\theta
\end{array}\right.
$$

\section{State Estimation Algorithm Based on EKF}

Mathematical model of wave-piercing catamaran longitudinal motion control system is a continuous time-varying nonlinear system, thus its discrete model is needed to get random state estimator design. And so, firstly the continuous time-varying nonlinear system should be discretized, the mathematical model of wave-piercing catamaran longitudinal motion control system is considered as the following form:

$$
\hat{\alpha}=f(\boldsymbol{x}, \boldsymbol{u}, t)+\boldsymbol{w}(t)
$$

Where $\boldsymbol{w}(t)$ is system random noise.

Extended Kalman filter (EKF) realize the linearization of nonlinear system function by one order Taylor expansion at the neighborhood of the state estimation point, the discretized model of nonlinear system is,

$$
\left\{\begin{array}{l}
\boldsymbol{x}_{k+1}=f\left(\boldsymbol{x}_{k}, \boldsymbol{u}_{k}\right)+\boldsymbol{w}_{k} \\
\boldsymbol{z}_{k}=h\left(\boldsymbol{x}_{k}\right)+\boldsymbol{v}_{k}
\end{array}\right.
$$

Nonlinear system function is one order Taylor expanded at the neighborhood of $\hat{\boldsymbol{x}}_{k}$, and second-order or higher order terms are ignored in order to realize the linearization of the nonlinear function. Also, the state of nonlinear system which has been approximately linearized still follows the gauss distribution, then you can use the full set of Kalman filter equations to design random state estimation for wave-piercing catamaran longitudinal motion control system.

$f\left(\boldsymbol{x}_{k}, \boldsymbol{u}_{k}\right)$ is one order Taylor expanded at the neighborhood of $\hat{\boldsymbol{x}}_{k}$, and the second and higher order terms are ignored,

$$
f\left(\boldsymbol{x}_{k}, \boldsymbol{u}_{k}\right)=f\left(\hat{\boldsymbol{x}}_{k}, \boldsymbol{u}_{k}\right)+\left.\frac{\partial f}{\partial \boldsymbol{x}}\right|_{\hat{\boldsymbol{x}}_{k}} \cdot\left(\boldsymbol{x}_{k}-\hat{\boldsymbol{x}}_{k}\right)
$$

$h\left(\hat{\boldsymbol{x}}_{k}\right)$ is one order Taylor expanded at the neighborhood of $\hat{\boldsymbol{x}}_{k \mid k-1}$, and the second and higher order terms are ignored,

$$
h\left(\boldsymbol{x}_{k}\right)=h\left(\hat{\boldsymbol{x}}_{k \mid k-1}\right)+\left.\frac{\partial h}{\partial \boldsymbol{x}}\right|_{\hat{\boldsymbol{x}}_{k \mid k-1}} \cdot\left(\boldsymbol{x}_{k}-\hat{\boldsymbol{x}}_{k \mid k-1}\right)
$$


Then the system model after approximate linearization becomes:

$$
\left\{\begin{array}{l}
\boldsymbol{x}_{k+1}=\phi_{k} \boldsymbol{x}_{k}+M_{k}+\boldsymbol{w}_{k} \\
z_{k}=C_{k} \boldsymbol{x}_{k}+N_{k}+\boldsymbol{v}_{k}
\end{array}\right.
$$

Traditional Kalman filter equations are arranged to series of EKF formula of nonlinear systems (6).

$$
\begin{gathered}
\hat{\boldsymbol{x}}_{k+1 \mid k}=f\left(\hat{\boldsymbol{x}}_{k}, \boldsymbol{u}_{k}\right) \\
\hat{\boldsymbol{z}}_{k+1}=h\left(\hat{\boldsymbol{x}}_{k+1 \mid k}\right) \\
\hat{\boldsymbol{x}}_{k+1}=\hat{\boldsymbol{x}}_{k+1 \mid k}+K_{k+1}\left(z_{k+1}-\hat{\boldsymbol{z}}_{k+1}\right) \\
P_{k+1 \mid k}=\phi_{k+1} P_{k} \phi_{k+1}^{\mathrm{T}}+Q_{k+1} \\
K_{k+1}=P_{k+1 \mid k} C_{k+1}^{\mathrm{T}} \cdot\left(C_{k+1} P_{k+1 \mid k} C_{k+1}^{\mathrm{T}}+R_{k+1}\right)^{-1} \\
P_{k+1}=\left(I-K_{k+1} C_{k+1}\right) P_{k+1 \mid k}
\end{gathered}
$$

It is known from formula (10) (15) that $M_{k}$ and $N_{k}$ don't directly appear in the formula. the basic process of EKF algorithm is: $\hat{\boldsymbol{x}}_{k+1 \mid k}$ and $\hat{\boldsymbol{z}}_{k+1}$ at the time of $k+1$ can be forecasted by the estimation of $\hat{\boldsymbol{x}}_{k}$ at the time of $k$, then $P_{k+1 k}$ is calculated, and predictive value and covariance matrix of the system state can be corrected based on the measured value $z_{k+1}$ at the time of $k+1$, and the system state $\hat{\boldsymbol{x}}_{k+1}$ and $P_{k+1}$ at the time of $k+1$ can be obtained. ${ }^{[6][7]}$

\section{Simulation Verification}

In order to verify the effectiveness of the proposed algorithm, simulation verification is performed for wave-piercing catamaran longitudinal motion control system. Before simulation study, firstly state equation and observation equation of wave-piercing catamaran longitudinal motion control system is discretized by using fourth-order Runge-Kutta method. Simulation speed is selected as $30 \mathrm{kn}$ and $35 \mathrm{kn}$; Simulation sea conditions is selected as significant wave height of $3 \mathrm{~m}$ and suffered waves to angle $60^{\circ}$, simulation results are shown in Figure 2 to 4 .
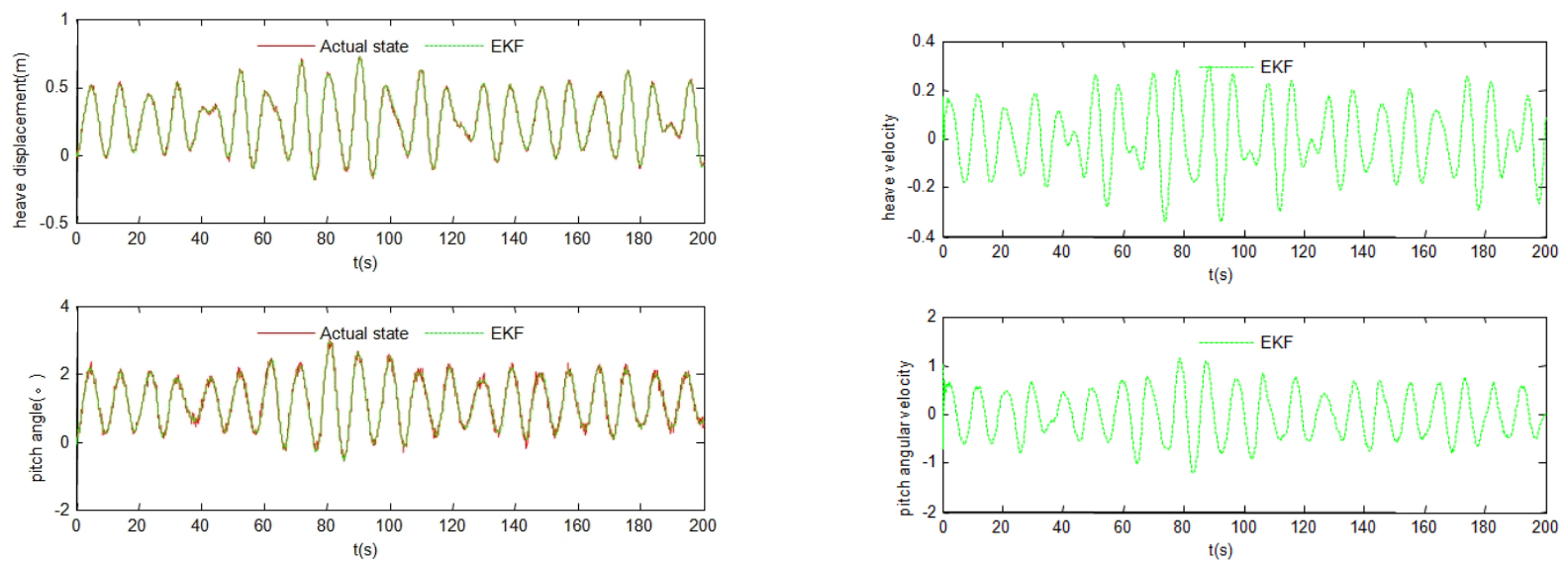

Fig. 2 Filtering results based on the EKF filtering algorithm

Fig.3 State estimation results 

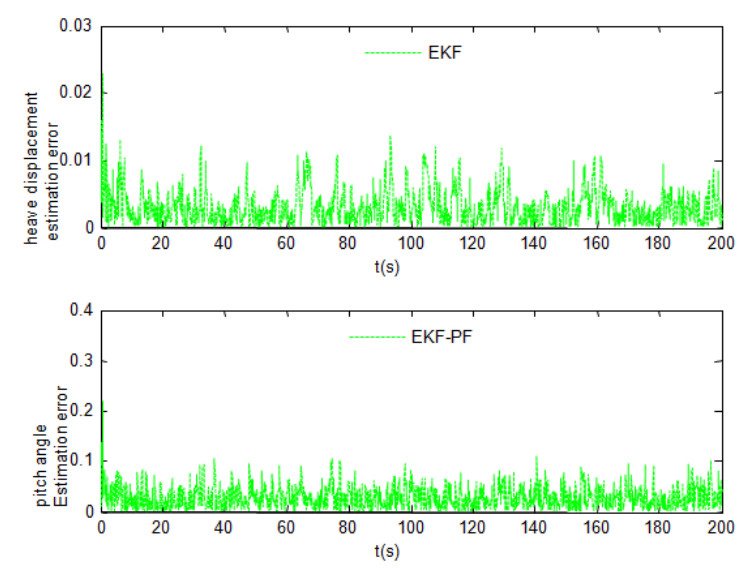

Fig.4 Filtering error based on the EKF filtering algorithm

\section{Summary}

For the characteristics of wave-piercing catamaran longitudinal motion when sailing with high speed in random waves, this paper proposes a state estimation algorithm for wave-piercing catamaran longitudinal motion based on EKF. Firstly, the wave-piercing catamaran longitudinal motion mathematical model is changed into the state space equation, then EKF algorithm is proposed, On the one hand noise is filtered out from the noise pollution measurement signal as much as possible, and the desired signal is separated therefrom; on the other hand, all state information of the system can be obtained by simply selecting the pitch angle and heave displacement of wave-piercing catamaran. The effectiveness of the proposed algorithm is verified through analyze of theory simulation research, and it provides technical support for the design and engineering practice of wave-piercing catamaran longitudinal motion control system.

If you follow the "checklist" your paper will conform to the requirements of the publisher and facilitate a problem-free publication process.

\section{References}

[1] Wencai Dong, Xiang Xia, Wenqiang Zuo. Experimental study on the performance of the light wave-piercing catamaran [J]. Shipbuilding of China, 2008, 49(3): 118-126.

[2] Lianen Zhao, Yonghe Xie.Principle and design of high-performance ship [M]. Beijing: National Defense Industry Press, 2009: 61-100.

[3] P.D.Samuel,D.Kutluil. Some Applications of Tensor Algebra to Estimation Theory[C]. The 3rd International Symposium on Wireless Pervasive Computing, 2008, 106-110.

[4] S. Luca. Optimal Estimation in Networked Control Systems Subject to Random Delay and Packet Drop[J]. IEEE Transactions on Automatic Control, 2008, 53(5): 1311-1317.

[5] Junsheng Ren, Yansheng Yang. Motion Modeling and Simulation of high-speed hydrofoil wave-piercing catamaran in Static water [J]. Journal of Dalian Maritime, 2002, 28(2): 18-21.

[6] Liangqun Li, Hongbing Li, Junhui Luo. Iterated extended Kalman particle filter [J]. Journal of Xidian University, 2007, 34(2): 233-238. 